PAPER • OPEN ACCESS

\title{
Spray measurements with the time-shift technique
}

To cite this article: Walter Schaefer et al 2021 Meas. Sci. Technol. 32105202

View the article online for updates and enhancements.

The Electrochemical Society

Advancing solid state \& electrochemical science \& technolog 2021 Virtual Education

\section{Intensive Short Courses}

Sun, Oct 10 \& Mon, Oct 11

Providing students and professionals with in-depth education on a wide range of topics

Early registration deadline: Sep 13, 2021 


\title{
Spray measurements with the time-shift technique
}

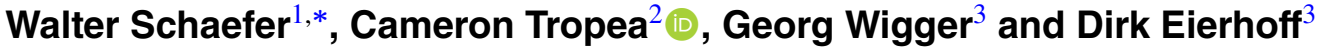 \\ ${ }^{1}$ AOM-Systems GmbH, Benzstr. 4, 64646 Heppenheim, Germany \\ ${ }^{2}$ Institute of Fluid Mechanics and Aerodynamics, Technische Universität Darmstadt, Alarich-Weiss-Str. \\ 10, 64287 Darmstadt, Germany \\ ${ }^{3}$ BASF Coatings GmbH, Glasuritstr. 1, 48165 Münster, Germany \\ E-mail:ws@aom-systems.com
}

Received 17 February 2021, revised 4 May 2021

Accepted for publication 24 May 2021

Published 22 June 2021

\begin{abstract}
The time-shift technique is a well-documented technique for the size and velocity measurement of individual drops passing through one or two tightly focused laser beams. It is a counting technique, nominally applicable for pure liquid drops, but with potential to also characterize drops with embedded particles or drops containing a second dispersed phase. In the present study a novel approach to signal processing is introduced in which the signal detection and validation phase is eliminated. This extends the capabilities of the time-shift technique in two manners. For one, size and velocity estimates are made possible for drops exhibiting very poor signal structure or signal-to-noise ratio. Such signals are commonly expected when measuring complex drops, either drops with embedded nano/micro-particles (dispersions) or emulsions. Second, the size and velocity distributions are estimated not by processing of signals from individual drops (single realization counting technique), but from a large ensemble of drop signals, improving both computational speed and reducing the influence of outliers in final statistics. These capabilities are achieved without sacrificing accuracy of mean and variance estimates of size and velocity of drop ensembles. To demonstrate the advantages of this new approach, measurements of a paint spray are presented, processed using both standard processing routines and the new approach. Limitations concerning the application of this new approach are discussed in detail.
\end{abstract}

Keywords: time-shift technique, sprays, drop size distribution, atomization

(Some figures may appear in colour only in the online journal)

\section{Introduction}

The time-shift technique is a counting technique for the measurement of drop size and velocity in a spray. It has first been introduced by [1] and more generally discussed in [2]. The two-beam time-shift technique is one realization, whereby the velocity is measured using a time-of-flight measurement [3].

* Author to whom any correspondence should be addressed.

Original Content from this work may be used under the terms of the Creative Commons Attribution 4.0 licence. Any further distribution of this work must maintain attribution to the author(s) and the title of the work, journal citation and DOI.
The use of two beams not only enables a time-of-flight velocity measurement, but increases the reliability of the size measurement due to redundancy used in the validation step. Further advances of the technique have been introduced to measure also the concentration of a dispersed phase in the drops [4] or the existence of embedded particles [5]. The former is encountered in processes like spray drying, the latter in metallic paint sprays.

However, there are many circumstances when the validation rate of the time-shift signals decreases to unacceptably low levels, resulting in statistics built on only a fraction of the detected drops in a spray and/or including outlier values. Such situations arise for instance in dense sprays, where secondary scattering leads to additional signal noise or when the 


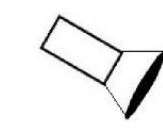

Signal Detector 1

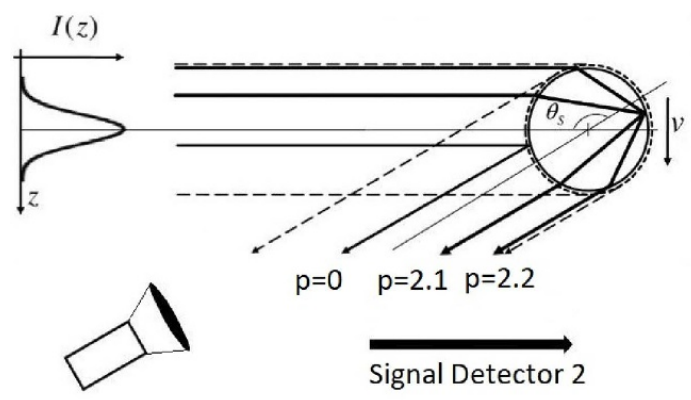

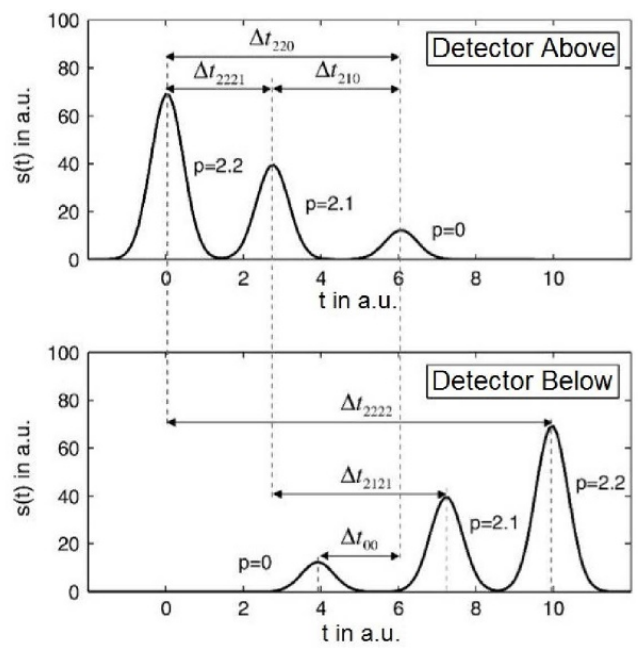

Figure 1. Time-shift measurement principle. Left diagram indicates main scattering orders contributing to the scattered light in backscatter (dashed lines represent surface waves). The signals registered by the two detectors are shown schematically in the two diagrams on the right. The various time shifts, denoted $\Delta t$ are directly proportional to drop diameter $d$ and inversely proportional to the velocity of drop passing through the illuminating beam, $v$.

embedded dispersed phase leads to highly distorted signals. The present study addresses this problem by eliminating the signal detection step and abandoning the counting approach, i.e. the evaluation of the signals from each individual drop, and reverting to the processing of a large ensemble of drop signals in an integral manner. While this approach no longer offers measurement data corresponding to each individual drop, it nevertheless yields robust and estimators of size and velocity distributions prevailing in the spray. Thus, this new processing approach extends the applicability of the time-shift technique to the measurement of denser sprays and to sprays of more complex drops, while reducing computational effort. The question to be addressed in this study is to what extent such estimators will be biased.

A brief introduction to the principles and implementation of the time-shift technique is first given to establish the signal form expected under ideal conditions. This is followed by a description of conventional signal processing algorithms, since results obtained using these algorithms will be used as a reference and comparison to results obtained using the new integrating approach. This comparison will provide information about the expectation of the integral estimators. The integrating approach, based on autocorrelation and crosscorrelation functions will then be introduced. Finally, sample measurement results will be presented to demonstrate the robustness of the new approach.

\section{Principles and implementation of the time-shift technique}

The time-shift principle is based on the fact that the particle to be measured-in this case drops-scattering light from an illuminating light beam, is considerably larger than the light beam waist diameter, $\omega_{0}$. While coherence and/or polarization is not necessary, time-shift instruments use lasers because of the necessity to tightly focus the beam at relatively long working distances (WDs). The working principle is illustrate in figure 1 . The fact that the illuminating beam, shown in this figure on the left-hand side with a Gaussian intensity distribution, is smaller than the particle of diameter $d$ passing through it with the velocity $v$ means that the individual scattering orders falling onto a detector are separated in time. In this figure detectors are shown in the near backscatter direction, where the following scattering orders are expected to contribute to the signal: reflection $(p=0)$, second-order refraction, which exhibits two unique paths (modes) through the drop ( $p=2.1, p=2.2)$, as well as surface waves on a long and short path, but which are much lower in amplitude and will be neglected in this study. Here, $p$ denotes the number of trajectories the light ray passes through the drop before exiting the drop toward the detector. The contribution of individual scattering orders to the signal can be obtained by applying a Debye decomposition [6] to the Lorenz-Mie solution of the light scattering from a sphere [7]. However, for simulations of the expected time-shift signals it is usually sufficient to use a paraxial geometric optics approximation [8] to compute the relevant scattering order contributions. It is noted here that the primary rainbow arises from interference between the second-order refractive scattering modes $p=2.1$ and $p=2.2$; however, since these modes are now separated in time on the detector, no interference phenomenon arises. This would only occur for drops of diameter comparable to the illuminating beam diameter, in which case the individual signal peaks begin to merge together. Furthermore, the detectors for the time-shift realization are generally not positioned where the primary rainbow is expected.

It is immediately apparent that the time shift between different scattering order/mode peaks in the signal either on one detector or between detectors will be linearly proportional to the diameter of the drop and inversely proportional to the velocity of the drop; hence, the term time shift. These various 

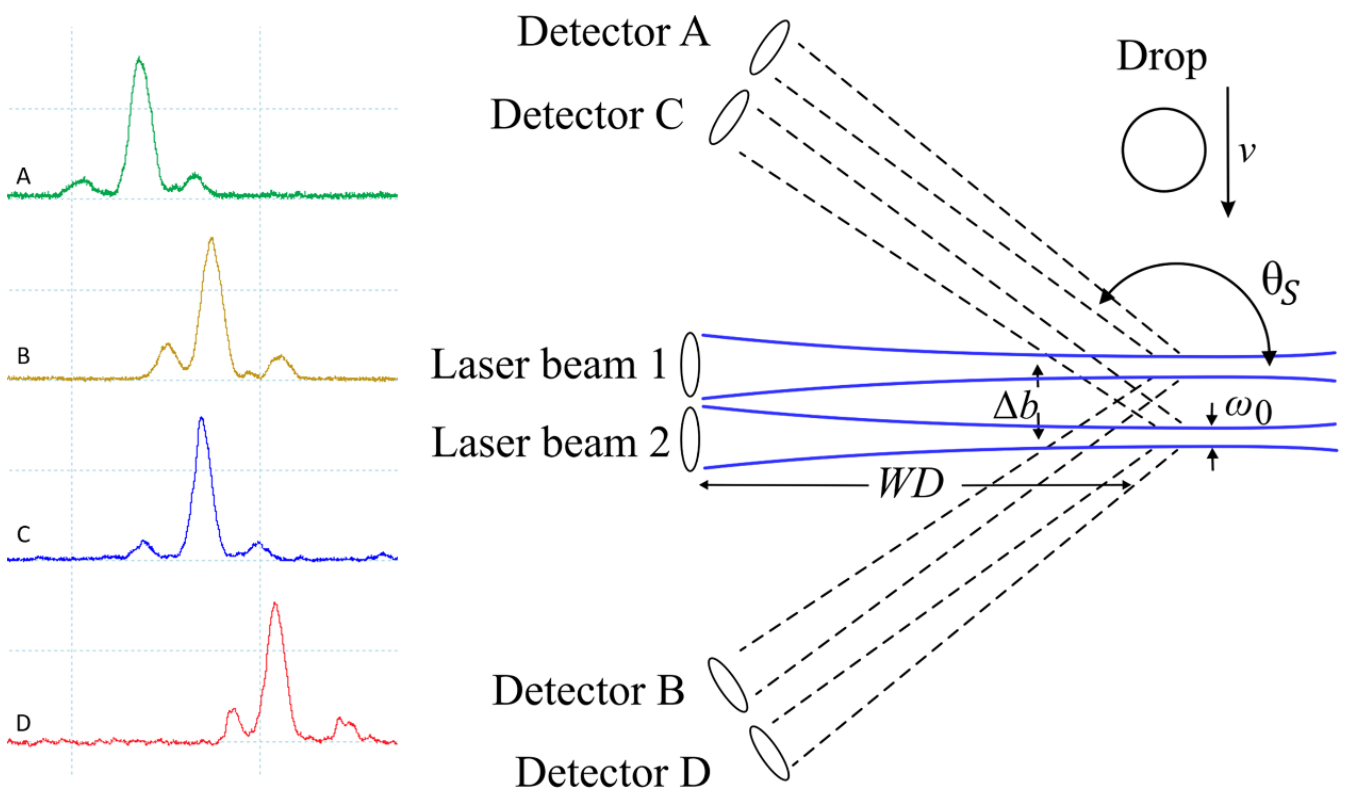

Figure 2. Schematic illustration of a two-beam time-shift optical configuration.

time shifts have been designated $\Delta t_{i j k l}$ in figure 1, where the subscripts $i, j, k$, and $l$ refer to the $p$ value of the respective scattering orders $(0,2.1$ or 2.2$)$. Thus, knowing a particular value $\Delta t$, a velocity measurement is necessary to determine the drop diameter. Also apparent is that there exists redundancy in the computation of drop diameter, since all of the various time shifts between signal peaks are linearly related to the drop diameter. Given the assumption of spherical particles/drops, the proportionality constants between each of the time shifts shown in the figure and the drop diameter will be given solely by the geometry of the optical configuration; hence, there is no need for calibration.

While numerous practical realizations of this measurement principle have been suggested, the most common technique is to use two illuminating beams aligned a distance $\Delta b$ downstream of each other in the main flow direction, thus enabling a time-of-flight (TOF) velocity measurement. Such an optical system is shown schematically in figure 2, showing four detectors in the near backscatter direction, two focused on each of the illuminating beams.

Designating the signals received on the four detectors pictured in figure 2 as $S_{A}, S_{B}, S_{C}$ and $S_{D}$, the time shift $\Delta t_{A C}$ between $S_{A}(t)$ and $S_{C}(t)$ and $\Delta t_{B D}$ between $S_{B}(t)$ and $S_{D}(t)$ are proportional to the droplet velocity and given by

$$
\Delta t_{A C}=\Delta t_{B D} \equiv \Delta t_{\mathrm{TOF}}=\frac{\Delta b}{v} .
$$

Thus, knowing the beam separation $\Delta b$, the velocity can be solved using equation (1), having measured the time shift between detectors $A$ and $C$ or $B$ and $D$, i.e. $\Delta t_{\text {TOF }}$. These two time shifts can be measured either using a cross-correlation or a peak detection algorithm in each of the respective signals, yielding two estimates of the drop velocity.
Size information is related to the time shifts between scattering orders, either within individual signals or between different signals. However, experience shows that the scattering order $p=2.1$ (second-order refraction, mode 1) dominates the signal at lower signal-to-noise ratios (SNRs) and for nonopaque drops/particles. For instance, the signals shown on the left of figure 2 are taken from a paint spray and the main peak corresponds to $p=2.1$. This provides two time shifts related to the drop diameter, the time shift between the main signal peak on detectors $A$ and $B$, or on detectors $C$ and $D$. This can be expressed as

$$
\Delta t_{A B}=\Delta t_{C D} \equiv \Delta t_{\mathrm{TS}}=\frac{d}{v} f\left(m, \theta_{s}, p\right)
$$

where $f\left(m, \theta_{s}, p\right)$ is a constant dependent on the relative refractive index of the drop (to the surrounding medium), $m$, the scattering angle at which the detector(s) is placed, $\theta_{s}$, and the scattering orders $p$ involved in the respective time shift. Substituting the two values of $v$ from equation (1) into the two possibilities given by equation (2) yields the following four expressions for the drop diameter

$$
\begin{aligned}
& d_{A B C}=\left(\frac{\Delta b}{f\left(m, \theta_{s}, p\right)}\right)\left(\frac{\Delta t_{A B}}{\Delta t_{A C}}\right) \\
& d_{A B D}=\left(\frac{\Delta b}{f\left(m, \theta_{s}, p\right)}\right)\left(\frac{\Delta t_{A B}}{\Delta t_{B D}}\right) \\
& d_{A C D}=\left(\frac{\Delta b}{f\left(m, \theta_{s}, p\right)}\right)\left(\frac{\Delta t_{C D}}{\Delta t_{A C}}\right) \\
& d_{B C D}=\left(\frac{\Delta b}{f\left(m, \theta_{s}, p\right)}\right)\left(\frac{\Delta t_{C D}}{\Delta t_{B D}}\right) .
\end{aligned}
$$


The function $f\left(m, \theta_{s}, p\right)$ is given as

$$
f\left(m, \theta_{s}, p\right)=\sin \left(\theta_{i}^{p=2.1}\right)
$$

where $\theta_{i}^{p=2.1}$ is the angle at which the $p=2.1$ ray enters the drop. This angle is given by the implicit equation [3]

$$
\sin \theta_{i}^{p=2.1}=m \sin \left(\frac{\pi}{4}-\frac{\theta_{s}}{4}+\frac{\theta_{i}^{p=2.1}}{2}\right) .
$$

The redundant values can be used to compute a mean and standard deviation for the size and velocity values for each drop (subscript $i$ ):

$$
\begin{gathered}
v_{i}=\langle v\rangle_{i} \pm \Delta v_{i}=\operatorname{mean}\left(v_{A C}, v_{B D}\right)_{i} \pm \operatorname{std}\left(v_{A C}, v_{B D}\right)_{i} \\
d_{i}=\langle d\rangle_{i} \pm \Delta d_{i}=\operatorname{mean}\left(d_{A B C}, d_{A B D}, d_{A C D}, d_{B C D}\right)_{i} \\
\pm \operatorname{std}\left(d_{A B C}, d_{A B D}, d_{A C D}, d_{B C D}\right)_{i} .
\end{gathered}
$$

The level of either standard deviation can be used as a validation criterion to either accept or reject the measurement. Given a large ensemble of single drop measurements in a spray, statistics regarding the size and velocity distribution at a point with relatively high spatial resolution can be obtained

$$
\bar{v}=\operatorname{mean}\left(v_{1}, \ldots, v_{N}\right) \pm \operatorname{std}\left(v_{1}, \ldots, v_{N}\right) \quad(68 \% \text { confidence })
$$

$$
\bar{d}=\operatorname{mean}\left(d_{1}, \ldots, d_{N}\right) \pm \operatorname{std}\left(d_{1}, \ldots, d_{N}\right) \quad(68 \% \text { confidence })
$$

where by $N$ indicates the number of the individual validated drops used for averaging. Thus, the time-shift technique operated in this manner can be considered a point measurement of single realizations. An implicit assumption to this approach of processing is that individual drop signals can be detected.

Signal detection in the presence of noise is a widely studied field, but has been specifically addressed for time-shift signals in [9]. Although in this theoretical study a single filter could be proposed with comparable detection performance of a matched filter bank, the detection power still falls off rapidly with decreasing SNR. The advances presented in the current study addresses the situation of low SNR in which signal detection becomes very difficult and time consuming.

A second implicit assumption in the above expressions is that the relative refractive index $(m)$ is constant. This is not strictly true, since different wavelengths of laser are used for the two illuminating beams to avoid any cross-talk in the detectors and the refractive indices of liquids are wavelength dependent. Typically the wavelengths $405 \mathrm{~nm}$ and $450 \mathrm{~nm}$ are used in commercial time-shift instruments for the two illuminating beams. However, for typical fluids, the resulting changes in $\theta_{i}^{p=2.1}$; hence, in $f\left(m, \theta_{s}, p\right)$, are negligible and are not further considered in this study.

Finally, it is noteworthy that the proportionality function $f\left(m, \theta_{s}, p\right)$ between time shift and size (equation (7)), is known entirely from the optical arrangement, i.e. no calibration of the instrument is necessary.

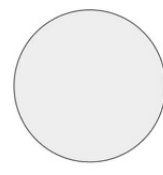

(a)

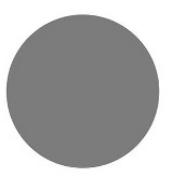

(b)

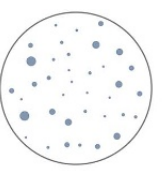

(c)

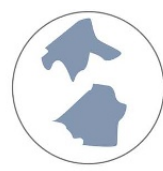

(d)

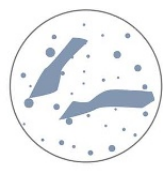

(e)
Figure 3. (a) Transparent drop, (b) opaque drop, (c) suspension/emulsion drop, (d) drop with embedded flakes and (e) suspension/emulsion droplet with embedded flakes.

\section{Signal processing}

\subsection{Signal distortion}

Strong signal distortions from the ideal forms shown in figure 1 occur when the drops are no longer spherical or are more complex in composition, examples of which were shown on the left side of figure 2. Examples of complex drops are given in figure 3, showing pure liquid drops-transparent and opaque-as well as drops containing a second dispersed phase or other particles/flakes. For such drops with embedded particles or emulsions, the $p=2.1$ contribution may undergo significant attenuation when passing through the drop. Furthermore, scattering from particles or inhomogeneities within the drop will lead to a background intensity level at the detectors, possibly completely obscuring the expected signal peaks. Moreover, if the drop is non-spherical the function $f\left(m, \theta_{s}, p\right)$ in equation (7) may no longer be amendable to theoretical derivation. Examples of theoretical solutions to the light scattering from non-spherical drops have been presented for specific shapes, e.g. spheroids [10]; however, the oblateness and drop orientation w.r.t. the illuminating beam must be known beforehand. Thus, an analytic solution for use with the randomness of drops passing through the detection volume of a time-shift instrument is not feasible.

Further to these causes of distortion, also the intensity of the various scattering orders will vary not only with embedded particle concentration in the drop, but also with the drop diameter. In the Mie scattering regime the intensity of scattered light varies approximately as

$$
I_{\mathrm{SCA}} \propto d^{2}
$$

and the intensity is expected to vary inversely with velocity

$$
I_{\mathrm{SCA}} \propto \frac{1}{v} .
$$

Lacking theoretical solutions of light scattering from such drops with complex composition, Monte Carlo ray-tracing approaches have been successfully used to simulate expected time-shift signals [4]. However, it is not practical to invoke a Monte Carlo simulation in some iterative manner on each and every of the four signals originating from a single drop to obtain an estimate of its velocity and size. The time shift between signals as shown in figure 2 is no longer uniquely defined and will now be representative of an average over the different scattering orders, weighted with their corresponding intensity (amplitude), all of which are quantitatively unknown. 


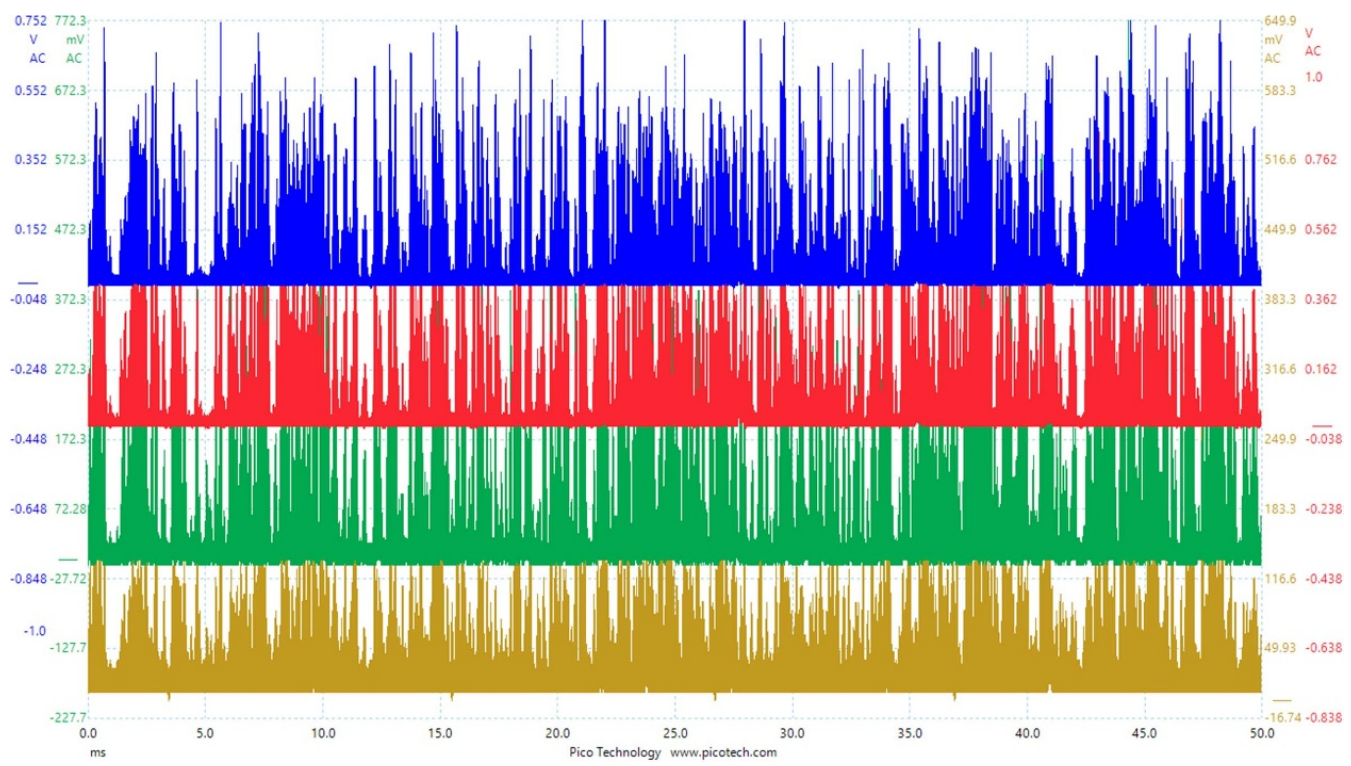

Figure 4. Time-shift signals from a water spray generated using a rotary bell atomizer with a rotation speed of $23000 \mathrm{rpm}$ and digitized at $312.5 \mathrm{MHz}$ over $50 \mathrm{~ms}$

Thus, signal processing according to equations (1) and (2) will not longer be directly applicable. The novel approach suggested here is therefore to derive velocity and size estimates based on the total ensemble of drop signals in an averaging manner based on autocorrelation and cross-correlation functions.

\subsection{Signal correlation approach}

An example extract of time-shift signals from the four instrument detectors is shown in figure 4 . This signal was taken from a water spray, generated using a rotary bell atomization operating at $23000 \mathrm{rpm}$ and digitized at a rate of $312.5 \mathrm{MHz}$ over $50 \mathrm{~ms}$. Approximately 400 single drops can be detected in this

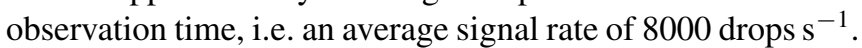
These signals fulfill the expectations of ideal signals and a single realization processing as described above can be carried out, yielding an average droplet velocity of $\bar{v}=17.0 \mathrm{~m} \mathrm{~s}^{-1}$ and average droplet size of $\bar{d}=43 \mu \mathrm{m}$. Water, being a non-opaque liquid, resulted in a strong dominance of the $p=2.1$ peak in the signals.

The autocorrelation and cross-correlation functions of these signals will now be considered. The autocorrelation function is defined as:

$$
R_{X X}(\tau)=\int_{0}^{T} S_{X}(t) S_{X}(t+\tau) d t
$$

and the cross-correlation function as:

$$
R_{X Y}(\tau)=\int_{0}^{T} S_{X}(t) S_{Y}(t+\tau) d t
$$

where the $X$ and $Y$ subscripts indicate which detectors are meant and $T$ is the time period of the measurement window. The autocorrelation and cross-correlation functions from the example signals shown in figure 4 are shown in figures 5 and 6, whereby the functions are only shown for

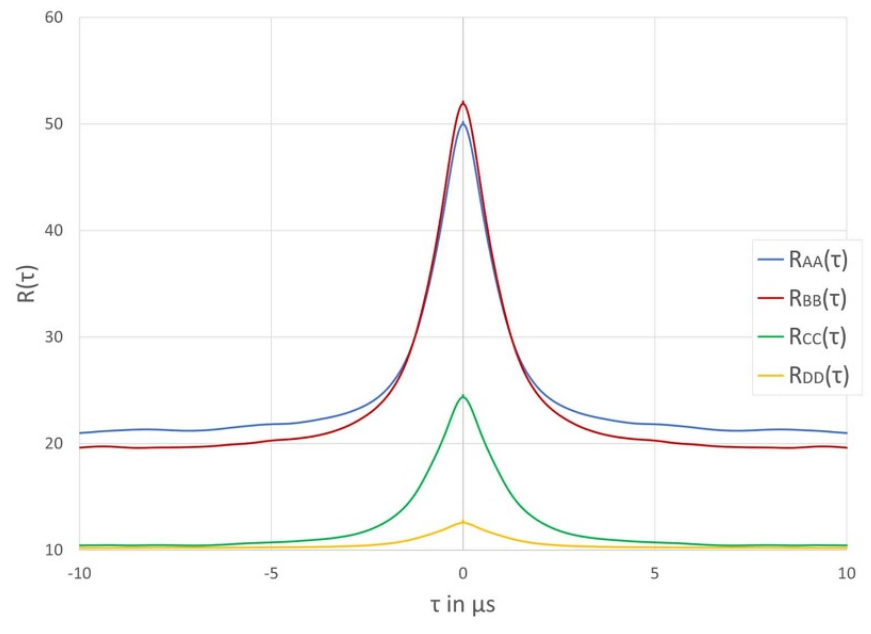

Figure 5. Autocorrelation functions $R_{A A}(\tau), R_{B B}(\tau), R_{C C}(\tau)$ and $R_{D D}(\tau)$ from the time-shift signals shown in figure 4 .

a range of lag times $\tau$ near the maximum value of the correlations.

The peak position of the autocorrelation function is by definition at $\tau=0$, whereby the amplitude of the autocorrelation function at $\tau=0$ yields the variance of the respective input signal over the time period $T$, including all noise contributions (sum of all self-products). This variance varies among detectors due to numerous factors related to the optical configuration and alignment, electronics and disturbing, yet uncontrollable factors, such as spray density. Nevertheless, leaving these factors constant, the variance will increase with signal amplitude and with the number of non-zero segments in a given observation time. Thus, the autocorrelation coefficient at $\tau=0$ is some measure of the number of drops appearing in this time window; hence, of the relative spray density. Unfortunately, because of the dependence also on signal amplitude, it is not 


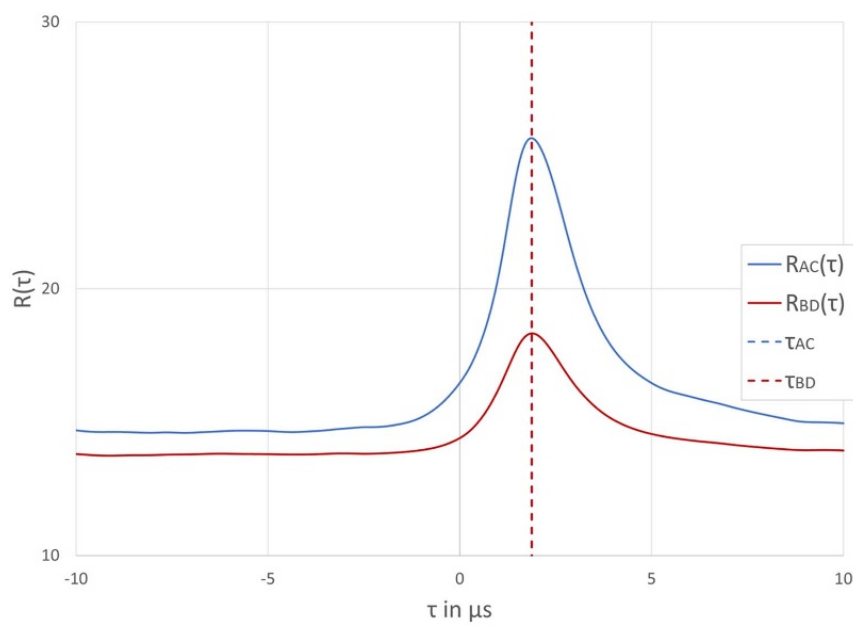

Figure 6. Cross-correlation functions $R_{A C}(\tau)$ and $R_{B D}(\tau)$ of the time-shift signals shown in figure 4 . The dashed line indicates the time lag of the maximum value of the cross-correlation functions. The time lag is used to compute the drop velocity using the time-of-flight approach.

straightforward to extract spray density from this measurement quantity - at most in a comparable manner. The shape of the autocorrelation function peak will be related to the average form of the individual peaks in the time-shift signals and may be revealing with respect to drop composition; however, this avenue of processing will not be further pursued in the present study.

Using a two-beam optical configuration, the time shift between detectors $A$ and $C$ or $B$ and $D$ will correspond to the time of flight of the drop between the two beams, irrespective of which scattering orders dominate the signal or how distorted the signals from an ideal form are. The lag time $\Delta \tau_{\max }$ of the cross-correlation maximum peak will yield an estimate of the average time-of-flight $\Delta t_{\mathrm{TOF}}$. The average velocity over all detected particles can therefore be estimated as

$$
\begin{aligned}
& \text { from } R_{A C}(\tau): \quad \bar{v}_{A C}=\frac{\Delta b}{\tau_{\max }} \\
& \text { from } \quad R_{B D}(\tau): \quad \bar{v}_{B D}=\frac{\Delta b}{\tau_{\max }} .
\end{aligned}
$$

The cross-correlation functions $R_{A C}(\tau)$ and $R_{B D}(\tau)$ from the signals in figure 4 are shown in figure 6 , in which the lag time $\tau_{\max }$ in each is clearly distinguishable, if not identical in either value or amplitude in the two cross-correlation functions. The average value of $\bar{v}_{A C}$ and $\bar{v}_{B D}$ computed from equations (17) and (18) can be used as a mean velocity estimate from all detected drops. Typical differences between these two velocities in the present experiments were of the order of $3 \%$.

The shape of the cross-correlation function can be considered to be a convolution of individual signal time shifts with the beam intensity shape, integrated over all drop signals, with a weighting proportional to respective scattering order strength. This can therefore be interpreted as a convolution of the beam shape with the velocity distribution the drop ensemble. However, retrieving the velocity distribution from

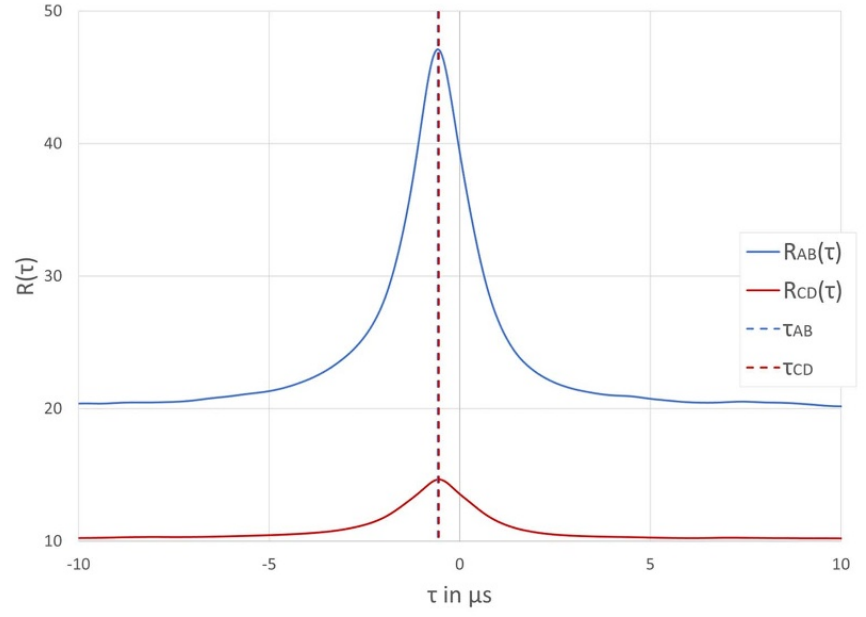

Figure 7. Cross-correlation functions $R_{A B}(\tau)$ and $R_{C D}(\tau)$ of the time-shift signals shown in figure 4 . The dashed line indicates the time lag of the maximum value of the cross-correlation functions. This time lag used used to compute the drop size.

the cross-correlation function remains difficult, since many influencing factors remain unknown, especially the uniqueness of the correlation between velocity and signal amplitude.

To estimate the mean drop size, equations (3)-(6) can be used, whereby the quantities $\Delta t_{A C}$ and $\Delta t_{B D}$ therein are estimated using the same $\tau_{\max }$ values from the $R_{A C}(\tau)$ and $R_{B D}(\tau)$ functions as used above for estimation of the velocity (equations (17) and (18)). The time shifts $\Delta t_{A B}$ and $\Delta t_{C D}$ in equations (3)-(6) can be estimated using the $\tau_{\max }$ values from the cross-correlation functions $R_{A B}(\tau)$ and $R_{C D}(\tau)$. These functions, computed from the signals in figure 4 , are shown in figure 7 , from which the respective $\tau_{\max }$ values are easily distinguishable, in this case negative. These values are negative, since the $p=2.1$ peaks dominate the signals and these peaks occur after the drop centerline has passed the beam centerline, i.e. at negative relative times.

The fact that the $p=2.1$ peak will dominate the signal is not self-evident. The relative amplitude of this peak will depend on the scattering angle, the absorption of light within the drop (imaginary part of the relative refractive index) and also any internal scattering within the drop. Hence, the $p=2.1$ peak amplitude will also depend on drop size. This effect of size dependent absorption and scattering will be discussed below in interpreting the measurement results.

In computing the size in this manner according to equations (3)-(6), the implicit assumption is made that the mean of $d / v$ is equal to the mean of $d$ divided by the mean of $v$, i.e.

$$
\left\langle\frac{d}{v}\right\rangle=\frac{\langle d\rangle}{\langle v\rangle} .
$$

This assumption will be valid if a strong correlation between size and velocity exists. This is often the case in sprays. For instance spraying into quiescent air will result in a positive correlation between size and velocity, or spraying into a much faster airflow will result in a negative correlation. As an 

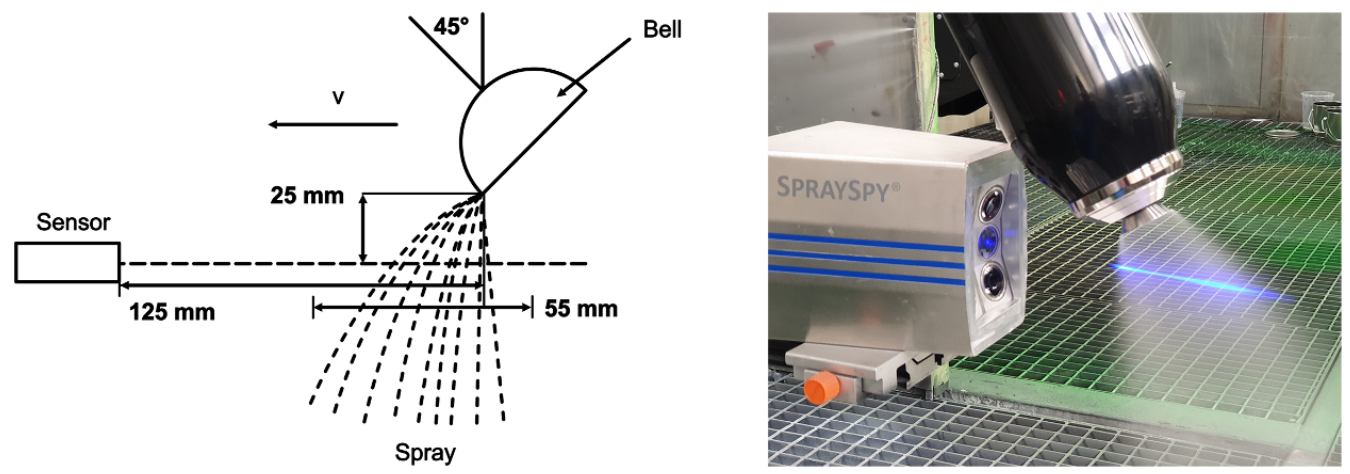

Figure 8. Schematic of experimental facility [11] and example photograph of the time-shift instrument. Note that the two beams and the four detectors shown in figure 2 are represented in this schematic simply by the dotted line and the sensor head.

example, in the latter case the smaller drops with less mass, will accelerate more rapidly; hence, small drops will have a higher velocity and large drops a lower velocity - a negative correlation. In this particular study, the validity of this assumption can be directly assessed, since measurements performed using the counting technique yield size and velocity for all drops and these data will be graphically presented in section 5, from which a strong correlation between the two quantities can be observed (see figures 9 and 10).

Also using this integration approach two estimates of velocity and four estimates of size are available and a mean and variance for each quantity can be computed, analog to equations (9) and (10). These averages will be denoted $v_{\Sigma}$ and $d_{\Sigma}$, indicating that these estimates are over the ensemble of drops as a whole.

\section{Experimental setup and conditions}

The experimental setup used in this study is the same facility reported on in [11], where the spray has been created by a rotary bell atomizer. A schematic illustration and an example photograph of the atomizer and instrument placement to measure the spray is shown in figure 8 . Note the tilt of the rotary atomizer to insure that the main flow direction of drops is aligned with the two detection volumes of the timeshift sensor. The operational parameters of the two conditions studied are summarized in table 1 . The two operational conditions vary primarily in the liquid atomized, in one case pure water and in the other black paint. The black paint is a colloidal drop with suspended, opaque nanoparticles, as depicted in figure 3(c).

The position of the measurement point is varied by traversing the nozzle at a constant speed and keeping the time-shift sensor stationary. Signals are acquired during the entire atomizer traverse, although no attempt is made to measure atomizer position with time, so it is not possible to assign any single drop measurement to a particular position in the spray. However, this is not necessary within the scope of the present study. The goal here was to measure a representative ensemble of drops throughout the entire spray.

The time-shift signals have been acquired using an external high speed digitizer (PicoScope 6404D) operated with a
Table 1. Operational parameters of both experimental conditions.

\begin{tabular}{lcc}
\hline Material & Flow rate & Rotational speed \\
\hline Water & $400 \mathrm{~nL} \mathrm{~min}^{-1}$ & $23000 \mathrm{rpm}$ \\
Black paint & $300 \mathrm{~nL} \mathrm{~min}^{-1}$ & $23000 \mathrm{rpm}$ \\
\hline
\end{tabular}

Table 2. Standard deviation in velocity and size using the counting approach of signal processing.

\begin{tabular}{lccc}
\hline Material & $\left\langle\frac{\Delta v}{\bar{v}}\right\rangle$ & $\left\langle\frac{\Delta d}{\bar{d}}\right\rangle$ & $\langle N\rangle$ \\
\hline Water & $8.0 \%$ & $19.9 \%$ & 181 \\
Black paint & $4.5 \%$ & $24.2 \%$ & 285 \\
\hline
\end{tabular}

sampling frequency of $321.5 \mathrm{MS} \mathrm{s}^{-1}$. The buffer of the digitizer allows to record up to $50 \mathrm{~ms}$ without losing samples. This yields approximately $1.6 \times 10^{6}$ samples per channel. In total 54 traverses have been performed, resulting in 54 data sets. The data sets M1-M13 were acquired for water and M14-M54 for black paint.

\section{Measurement results}

The acquired data sets were processed using the two approaches mentioned above; the conventional counting approach described in section 2 and using the cross-correlation functions, described in section 3.2. Since the cross-correlation approach only yields integral estimates, a direct comparison of the results from the two approaches is only possibly after averaging the values obtained using the counting approach.

The average number of the single drops used in the counting approach is given in table 2, where the dimensionless standard deviation of both the velocity and size values is shown. These values have been averaged over the 13 and 41 measurement data sets for water and black paint respectively. The standard deviation provides some indication of the data scatter involved in these measurements.

The velocity calculation can be expected to be of higher accuracy than the drop size calculation, because the calculated 


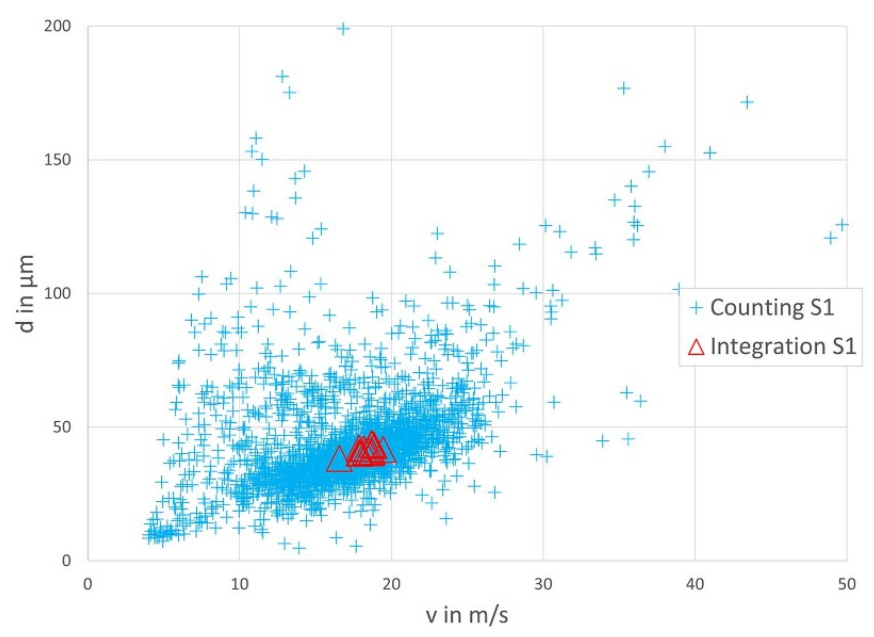

Figure 9. Measurement results obtained for experimental conditions S1 (water). The cross symbols indicate the results obtained using the counting method. The triangular markers indicate results obtained using the cross-correlation functions.

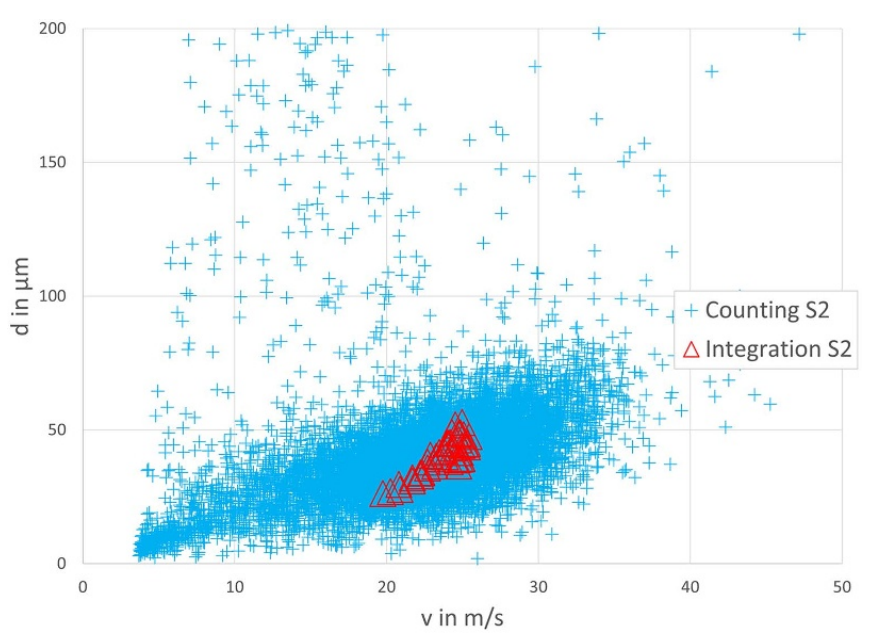

Figure 10. Measurement results obtained for experimental conditions S2 (black paint). The cross symbols indicate the results obtained using the counting method. The triangular markers indicate results obtained using the cross-correlation functions.

drop velocity is derived from a single time-shift value (see equation (1)), whereas the drop size is computed using two time-shifts (see equations (3)-(6)).

The measurement data from both processing methods are compared in figure 9 for measurements corresponding to the conditions S1 (water) and in figure 10 for conditions S2 (black paint). In these figures the size and velocity of each individual drop over all measurement campaigns (M1-M13, M14-M54) have been included. It is apparent from these plots that the scatter of measurements using black paint, especially in the size determination, is larger.

To further compare these signal processing approaches, the mean values of size and velocity from the counting approach is plotted against the results obtained using the cross-correlation approach in figures 11 and 12. Perfect agreement between the two approaches would correspond to the dashed line shown

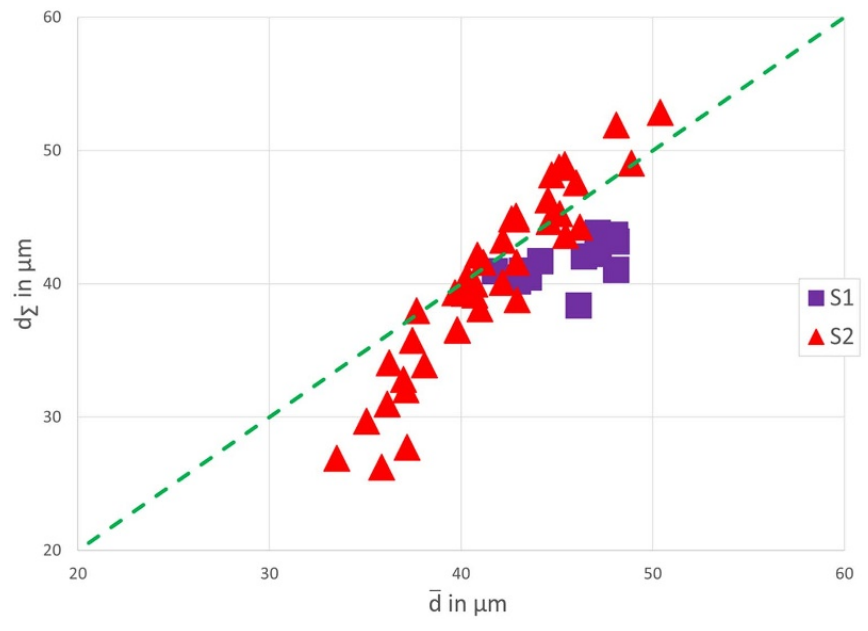

Figure 11. Comparison between measured mean drop size using counting $(\bar{d})$ and integration $\left(d_{\Sigma}\right)$ methods for water (S1) and black paint (S2).

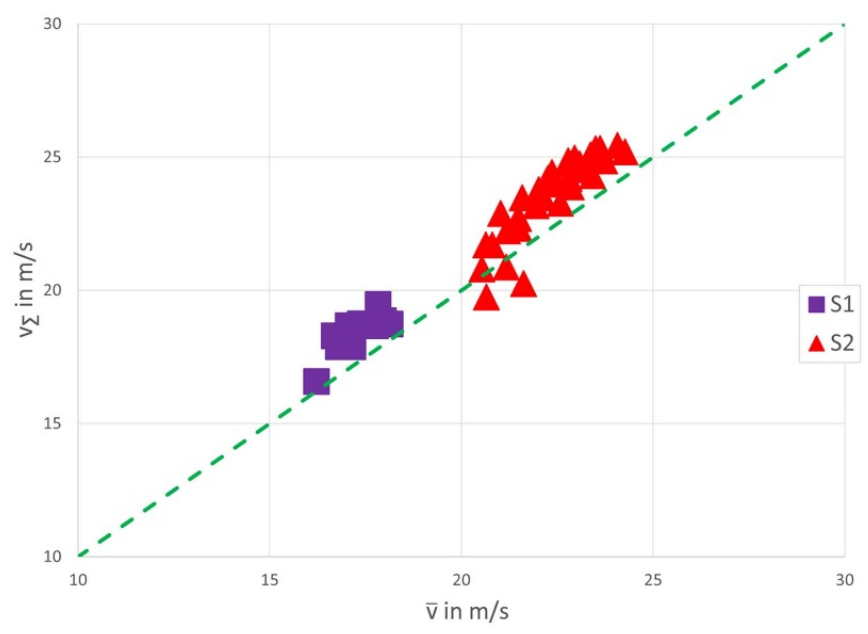

Figure 12. Comparison between measured mean velocity using counting $(\bar{v})$ and integration $\left(v_{\Sigma}\right)$ methods for water (S1) and black paint (S2).

in these figures. It is evident that deviations exist between the processing methods for both the measurements of water drops and those of black paint.

For water drops the correlation approach yields consistently smaller average drop sizes (figure 11). Whereas the conventional processing approach determines the size from the time shift alone from the time shift of the second-order refractive peaks $(p=2.1)$, the shift of the cross-correlation peak is biased towards a lower value by the contributions of the reflective peak in the input signals. This shift of the lag time toward lower values results in lower size estimates, according to equations (3)-(6).

For black paint the time-shift measurement is based on the reflective signal peak $(p=0)$, since the second-order refractive peak $(p=2.1)$ is highly attenuated. This holds true for large drops, since the refractive rays are completely absorbed during their passes through the drop. However, as the drops become smaller, the second-order refractive rays increase in intensity 
at the detector. This contribution to the detector signal then shifts the cross-correlation peak towards lower values, resulting in the lower values of mean drop size for the correlation method. The difference between the mean drop size measured using the two methods will be primarily dependent on the absorption degree of the colloidal drop. Hence, knowing the drop size, an estimate of the absorption coefficient of the liquid can be made, indicating some measure of concentration and/or size of the suspended nanoparticles. However, this possibility of estimating colloidal concentration will not be pursued, but has been investigated in a separate study [12].

As with the mean diameter measurement, the velocity measurements also exhibit a clear decrease of mean velocity for the correlation approach. The data points associated with these lower values correspond also to the data points exhibiting lower mean diameters. Again, for small black paint drops, the $p=2.1$ peak in the signal becomes stronger and biases the cross-correlation function to lower values of of the time shift.

\section{Summary and conclusions}

This study has introduced a novel signal processing approach for estimating size and velocity from time-shift signals. The novel approach is based on cross-correlation functions between the four detector signals with the intention of reducing computational effort and allowing measurements to be performed, despite lower signal-to-noise ratios arising due to unfavourable measurement conditions or due to the complex structure of drops. To evaluate the performance of these new estimators, comparisons were drawn to results obtained using a conventional, single realization approach to signal processing.

The results of the study indicate that the novel signal processing approach is viable, but exhibits certain application limits. The most important of these is that a single scattering order dominates the time-shift signal. For non-opaque drops (like from water), this would typically be second-order refraction $(p=2.1)$ and for opaque drops this would be reflection $(p=0)$. However, in the latter case and for very small drops, the signal contribution from second-order refraction can increase and bias both size and velocity estimates.

In the present study the results using the new integration approach could be compared with the conventional approach, revealing a lower size limit of about $40 \mu \mathrm{m}$. However this possibility is not always available and then the question arises whether this limit in size can be predicted prior to measurement, for instance using light scattering codes. For this, the complex relative refractive must be known, including the imaginary part responsible for absorption. Then a code capable of accounting for the Gaussian beam properties must be used, i.e. a code realizing the Generalized Lorenz-Mie Theory. However even then, the effect of decreased $p=2.1$ peak amplitude on the cross-correlation functions will not be unique, since many other factors come into influence, in particular noise. Thus, the lower size limit using this new technique is not straightforward to determine prior to measurement.
Preliminary measurements, as elaborated in the present study, are likely necessary and recommended.

Several avenues for future research have been revealed in this study. One is the possibility of extracting not just estimates of the mean size and velocity of a drop ensemble, but also extracting their distributions. This information is contained in the shape of the computed correlation functions. Another direction involves the estimate of the attenuation coefficient inside a drop (and thus colloidal concentration), which is related to the bias to the size estimate introduced by secondorder refractive scattering. For this a comparison to conventional estimates is necessary.

\section{Data availability statement}

The data generated and/or analysed during the current study are not publicly available for legal/ethical reasons but are available from the corresponding author on reasonable request. Data underlying the results presented in this paper are not publicly available at this time but may be obtained from the authors upon reasonable request. The data which can be made available are the raw digitized time-shift signals corresponding to the 54 data records M1-M54, an example of which is shown in figure 4.

\section{Funding}

No external funding sources were used to conduct this study.

\section{Disclosures}

The authors declare no conflict of interests, beyond their affiliation with AOM-Systems GmbH.

\section{ORCID iD}

Cameron Tropea (D) https://orcid.org/0000-0002-1506-9655

\section{References}

[1] Semidetnov N V 1985 Investigation of laser Doppler anemometer as instrument for two-phase flow measurements (in Russian) PhD Thesis Leningrad Institute for Precision Mechanic and Optics

[2] Albrecht H-E, Damaschke N, Borys M and Tropea C 2013 Laser Doppler and Phase Doppler Measurement Techniques (Heidelberg: Springer Science \& Business Media)

[3] Schäfer W 2013 Time-Shift Technique for Particle Characterization in Sprays (Berlin: epubli $\mathrm{GmbH}$ )

[4] Li L, Stegmann P G, Rosenkranz S, Schäfer W and Tropea C 2019 Simulation of light scattering from a colloidal droplet using a polarized Monte Carlo method: application to the time-shift technique Opt. Express 27 36388-404

[5] Li L, Rosenkranz S, Schäfer W and Tropea C 2019 Light scattering from a drop with an embedded particle and its exploitation in the time-shift technique J. Quant. Spectrosc. Radiat. Transfer 227 20-31 
[6] Debye P 1909 Der Lichtdruck an Kugeln von beliebige material Ann. Phys. 30 57-136

[7] Gouesbet G and Gréhan G 2011 Generalized Lorenz-Mie Theories vol 31 (Berlin: Springer)

[8] Glantschnig W J and Chen S-H 1981 Light scattering from water droplets in the geometrical optics approximation Appl. Opt. 20 2499-509

[9] Rosenkranz S, Schäfer W, Tropea C and Zoubir A M 2016 Modeling photon transport in turbid media for measuring colloidal concentration in drops using the time-shift technique Appl. Opt. 55 9703-11
[10] Xu F, Ren K, Gouesbet G, Gréhan G and Cai X 2007 Generalized Lorenz-Mie theory for an arbitrarily oriented, located and shaped beam scattered by a homogeneous spheroid J. Opt. Soc. Am. A 24 119-31

[11] Wigger G, Briesenick D, Eierhoff D, Bornemann C, Riediger S, Goedeke L and Ehrhard P 2020 Method for determining the droplet size distribution during atomization and screening method based thereon in paint development WO 2020/002245 A1

[12] Li L and Tropea C 2021 Measurement of the colloidal particle concentration and size within a drop using the time-shift technique J. Quant. Spectrosc. Radiat. Transfer 263107548 